02,09

\title{
Селективные измерения состояний сверхпроводникового кубита нелинейным джозефсоновским осциллятором
}

\author{
(C) В.П. Гергель, М.В. Денисенко , А.В. Линёв, А.М. Сатанин \\ Нижегородский государственный университет им. Н.И. Лобачевского, \\ Нижний Новгород, Россия \\ ฯ E-mail: mar.denisenko@gmail.com
}

\begin{abstract}
Изучается процесс селективных измерений состояний единичной квантовой системы - джозефсоновского кубита - нелинейным осциллятором, работающим в мезоскопическом режиме, когда число квантов в процессе измерения варьируется от нескольких десятков до нескольких сотен. Квантовым методом Монте-Карло промоделированы диссипативная динамика системы кубит-осциллятор и процесс измерений состояний кубита по изменению числа квантов осциллятора. Показано, что для $\pi$-импульсов записи состояний кубита возможно дискриминирование состояний в единичных реализациях (аналог „однократных“ измерений), а для приготовленного суперпозиционного состояния - проведение статистических проективных измерений.
\end{abstract}

Работа поддержана проектами РФФИ № 16-57-51045 НИФ а и 16-07-01012 а и стипендией Президента РФ для молодых ученых и аспирантов, осуществляющих перспективные научные исследования и разработки по приоритетным направлениям модернизации российской экономики в 2016-2018 г.

\section{1. Введение}

В последние годы разработаны различные методы измерений состояний джозефсоновских кубитов, например, такие как статистические проективные измерения [1,2], непрерывные неразрушающие измерения [3] (мониторинг системы в реальном времени) и т.п. В качестве измерительного прибора, в частности, может выступать джозефсоновский осциллятор, работающий в слабо нелинейном режиме вблизи точки бифуркации. К настоящему времени детально изучена связанная система кубит-осциллятор и разработан протокол неразрушающих измерений состояний кубита $[1,2]$, когда измерительный прибор является сугубо классической системой. В этом случае после инициализации состояния кубита осциллятор возбуждается из вакуумного состояния в состояние с большими числами заполнения $n$. В действительности кубит и осциллятор взаимодействуют, что приводит к „Перепутыванию“ их состояний и обратному воздействию измерительного прибора на кубит. Следовательно, модельная ситуация, рассмотренная в работах $[1,2]$, не учитывает факта перепутывания состояний, а также того, что измерительный прибор может работать в мезоскопическом режиме с большим, но конечным числом квантов. Таким образом, основной задачей настоящей работы является изучение вопроса о том, насколько квантовая система (кубит) чувствительна, а измеритель информативен в мезоскопическом режиме, например, когда среднее число квантов хотя и велико, но конечно, а среднеквадратичное отклонение мало по сравнению с числом заполнения.

Обычно для численного расчета релаксационной динамики квантовых систем используется подход, основанный на решении уравнения для матрицы плотности [4], который описывает поведение единичных квантовых систем только в среднем. Соответственно на основе этого метода нельзя исследовать динамику систем в каждой реализации (в одном акте измерения), наблюдать квантовые скачки, происходящие в системе за счет энергетической или фазовой релаксации, промоделировать процессы контроля квантовой системы в реальном времени - „однократные“ измерения. В связи с этим в настоящей работе расчет релаксационной динамики и моделирование процессов измерений состояний связанной системы кубит-осциллятор выполнены на основе квантового метода Монте-Карло [5]. Данный подход позволяет также промоделировать процесс накопления экспериментальной информации о единичной квантовой системе.

\section{2. Модель системы кубит-осциллятор}

Для моделирования процесса детектирования состояний квантовой системы - зарядового кубита специального вида („квантрониум“-кубита) [6] — в качестве примера измерительного устройства выбран джозефсоновский нелинейный осциллятор. Предполагается, что в качестве кубита выступают два джозефсоновских перехода с емкостью $C_{J}$ и определенными значениями джозефсоновской $E_{J} / 2$ и кулоновской $E_{C}$ энергий, функционирующие в режиме кулоновской блокады (рис. 1), которые соединены со сверхпроводящим островком (темный кружок, разделяющий джозефсоновские переходы на рис. 1) [3]. Используя уравнения Джозефсона и законы Кирхгофа, можно записать полный гамильтониан системы

$$
H=H_{Q Q}+H_{J},
$$

где гамильтониан расщепленного куперовского островка $H_{Q Q}$ имеет вид

$$
H_{Q Q}=4 E_{C}\left(\hat{N}-\frac{1}{2}+\frac{C_{g} V(t)}{2 e}\right)^{2}-E_{J} \cos \frac{\delta}{2} \cos \theta,
$$




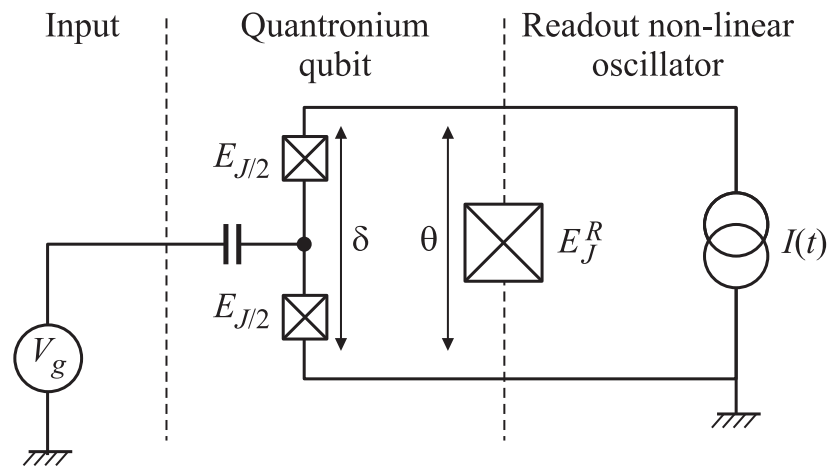

Рис. 1. Схема кубита (в центре рисунка) связанного с устройством записи (слева) и взаимодействующего со слабодиссипативным измерительным джозефсоновским осциллятором (справа). Перечеркнутыми квадратами обозначены джозефсоновские переходы. Островок (темный кружок, разделяющий перечеркнутые квадраты) является в то же время вторым контактом переходов (см. [1]).

а гамильтониан измерительного джозефсоновского осциллятора равен

$$
H_{J}=\frac{\hat{Q}^{2}}{2 C}-E_{J}^{R} \cos \theta-\frac{\hbar^{2}}{2 e} I(t) \theta,
$$

где $\hat{N}$ и $\hat{Q} / 2 e(e-$ заряд электрона $)-$ обобщенные импульсы, канонически сопряженные фазам $\delta$ и $\theta$ соответственно, куперовского и джозефсоновского переходов, характеризущихся энергиями $E_{J}$ и $E_{J}^{R}$ и емкостью $C$ шунтирующего большого джозефсоновского перехода (подробности вывода гамильтониана системы можно найти в работе [6]). Как видно, связь двух подсистем осуществляется за счет общего участка цепи, обусловливающего зависимость от фазы $\theta$ в потенциальной энергии выражения (1). Управление состояниями кубита осуществляется переменным напряжением $V(t)=V_{g}+V_{r f}(t) \cos \omega t$, которое меняет заряд на емкости $C_{g}$, а переменный ток $I(t)$ в правой части контура служит для управления измерительным джозефсоновским осциллятором.

Вблизи точки кулоновского вырождения, когда энергии островка для $N$ и $N+1$ частиц равны, можно использовать базисные состояния $|N\rangle$ и $|N+1\rangle(\hat{N}|N\rangle=N|N\rangle)$. В подпространстве $|N\rangle=(1,0)^{T}$ и $|N+1\rangle=(0,1)^{T}$ гамильтониан аппроксимируется выражением

$$
\begin{aligned}
H \cong & 2 E_{C} \frac{C_{g} V_{r f}(t)}{e} \sigma_{x}-E_{J} \cos \frac{\theta}{2} \sigma_{z}+\frac{Q^{2}}{2 C} \\
& -E_{J}^{R} \cos \theta-\frac{\hbar^{2}}{2 e} I(t) \theta
\end{aligned}
$$

где $\sigma_{x}$ и $\sigma_{z}-$ спиновые матрицы Паули.

При относительно малом токе $I(t)$ джозефсоновский осциллятор работает в слабо нелинейном режиме. То- гда потенциал в выражении (3) можно разложить по степеням $\theta$

$$
\begin{aligned}
H \cong & 2 E_{C} \frac{C_{g} V_{r f}(t)}{e} \sigma_{x}-\frac{E_{J}}{2} \sigma_{z}+\frac{Q^{2}}{2 C}+E_{J}\left(1+\lambda \sigma_{x}\right) \frac{\theta^{2}}{2} \\
& -E_{J}\left(1+\frac{\lambda}{4} \sigma_{z}\right) \frac{\theta^{4}}{4 !}-\frac{\hbar^{2}}{2 e} I(t) \theta
\end{aligned}
$$

где $\lambda=E_{J} / 4 E_{J}^{R}$.

Вводя операторы рождения $a^{\dagger}$ и уничтожения $a$ для параметризации координаты $\hat{\theta}=\left(a+a^{\dagger}\right)\left(2 E_{C}^{R} / E_{J}\right)^{1 / 4}$ и импульса осциллятора $\hat{N}=-i\left(a-a^{\dagger}\right)\left(E_{C}^{R} / 2 E_{J}\right)^{-1 / 4}$ $(\hat{Q}=2 e \hat{N}, \hat{N}=-i \partial / \partial \theta)$ с учетом коммутационного соотношения $[\hat{\theta} \cdot \hat{N}]=i$, можно эффективный гамильтониан (4) связанной системы кубит-осциллятор представить в виде

$$
H=H_{q}+H_{\text {osc }}+H_{\text {int }} .
$$

Здесь первое слагаемое - гамильтониан кубита $H_{q}=\frac{\hbar}{2}\left(\omega_{q} \sigma_{z}+\varepsilon(t) \sigma_{x}\right)$, где расстояние между уровнями кубита определяется джозефсоновской энергией $\hbar \omega_{q}=-2 E_{J}$, а управляющая функция $\varepsilon(t)=$ $=4 E_{C} C_{g} V_{r f}(t) / e$ индуцирует переходы между состояниями кубита $|\sigma\rangle\left(\sigma_{z}|\sigma\rangle=\sigma|\sigma\rangle, \sigma= \pm 1\right)$. Предполагается, что управление кубитом осуществляется Раби-импульсами переменного поля $\varepsilon(t)=A \cos \omega t$ определенной длительности.

Измерение состояний кубита предполагается осуществлять нелинейным осциллятором (4), который может быть переведен в когерентное состояние током „накачки“ $I(t)$ [2]. Гамильтониан измерительного осциллятора в терминах операторов рождения и уничтожения с учетом выражений для $\hat{\theta}=\left(a+a^{\dagger}\right)\left(2 E_{C}^{R} / E_{J}\right)^{1 / 4}$ и $\hat{Q}=-2 i e\left(a-a^{\dagger}\right)\left(E_{C}^{R} / 2 E_{J}\right)^{-1 / 4}$ имеет вид

$$
H_{\mathrm{osc}}=\hbar \omega_{J} a^{\dagger} a-\frac{\mu}{4}\left(a^{\dagger}+a\right)^{4}+f(t)\left(a+a^{\dagger}\right),
$$

где $\omega_{J}=\sqrt{\frac{2 e I_{c}}{\hbar C}}-$ собственная частота осциллятора $\left(I_{c}-\right.$ критический ток на большом джозефсоновском переходе), $\mu=E_{C}^{R} / 12 \hbar-$ параметр нелинейности, $f(t)=f_{0} \cos \Omega t$ - возбуждающее поле осциллятора $\left(f(t) \sim I(t) / I_{c}, f_{0}=\frac{\hbar}{2 e}\left(\frac{2 E_{C}^{R}}{E_{J}}\right)^{1 / 4}\right)$.

Наконец, последнее слагаемое в (5) отвечает за взаимодействие кубита с измерительным осциллятором

$$
H_{\mathrm{int}}=\lambda\left(\hbar \omega_{J} a^{\dagger} a-\frac{1}{4} \mu\left(a^{\dagger}+a\right)^{4}\right) \sigma_{z} .
$$

Отметим, что если управляющее поле отсутствует $(\varepsilon(t)=0)$, то оператор взаимодействия (7) коммутирует с гамильтонианом кубита $H_{q}$, поэтому осциллятор производит так называемое „неразрушающее измерение“ $[1,2,7]$ переменной $\sigma_{z}$. В случае же действия поля $(\varepsilon(t) \neq 0)$ состояния подсистем являются запутанными.

\section{3. Диссипативная динамика в рамках квантового метода Монте-Карло}

При изучении процесса детектирования состояний кубита учитываются процессы декогерентности, кото- 
рые можно представить как взаимодействие системы кубит-осциллятор с фононным резервуаром с большим числом степеней свободы [4], а вклад термического возбуждения при записи интеграла столкновения не рассматривается, так как эксперименты над сверхпроводниковыми кубитами проводятся при криогенных температурах [1-3]. Следовательно, гамильтониан шума, соответствующий вкладу бозонных возбуждений, имеет вид

$$
H_{\text {noise }}=F_{z} \sigma_{z}+F_{x} \sigma_{x}+R a^{\dagger}+R^{\dagger} a,
$$

где эрмитовы операторы $F_{z}(t), F_{x}(t)$ резервуара отвечают за продольную и поперечную релаксацию кубита, а оператор $R(t)$ - за релаксацию измерительного прибора.

Уравнение для оператора плотности $\rho$, где исключены переменные резервуара $F(t), R(t)$ и использовано приближение Борна-Маркова [4], записывается в виде

$$
\begin{aligned}
\frac{\partial \rho}{\partial t}= & \frac{1}{i \hbar}[H, \rho]+\frac{\Gamma_{\varphi}}{2}\left(\sigma_{z} \rho \sigma_{z}-\rho\right) \\
& +\frac{\Gamma_{e}}{2}\left(2 \sigma_{-} \rho \sigma_{+}-\sigma_{+} \sigma_{-} \rho-\rho \sigma_{+} \sigma_{-}\right) \\
& +\frac{\gamma}{2}\left(2 a \rho a^{+}-a^{+} a \rho-\rho a^{+} a\right),
\end{aligned}
$$

где матрицы $\sigma_{ \pm}=\left(\sigma_{x} \pm i \sigma_{y}\right) / 2$ определены стандартным образом через матрицы Паули $\sigma_{x}, \sigma_{y}, \sigma_{z}$, а $\Gamma_{\varphi}$ и $\Gamma_{e}-$ coответственно фазовая и энергетическая скорости релаксации кубита, $\gamma$ - параметр энергетической релаксации осциллятора. Отметим, что релаксационные параметры выражаются стандартным образом через корреляционные функции бозонного термостата $\left(\left\langle F_{z}(t) F_{z}^{+}\right\rangle,\left\langle R(t) R^{+}\right\rangle\right.$ и т.д.).

Динамику связанной системы кубит-осциллятор в единичных реализациях (в каждом акте измерения) опишем с точки зрения квантового метода Монте-Карло [5]. Для этого можно представить эволюцию связанной системы как динамику некоторой реализации чистого состояния $\rho_{i}(t)=\left|\phi_{i}(t)\right\rangle\left\langle\phi_{i}(t)\right|$ (индекс $i$, нумерующий единичные реализации, будем опускать в тех местах, где это не вызывает недоразумений). В этом случае стохастическая волновая функция связанной системы $\left.\left|\phi_{i}(t)\right\rangle=\sum_{n, \sigma} C_{i, n, \sigma}(t) n \sigma\right\rangle$ может быть разложена по базису $|n \sigma\rangle=|n\rangle \otimes|\sigma\rangle$, где $\otimes$ означает тензорное произведение фоковского базиса линейного осциллятора $|n\rangle$ и собственного базиса кубита $|\sigma\rangle$. Следовательно, состояние системы описывается $2 N$-компонентной стохастической волновой функцией, где $N$ - число уровней нелинейного осциллятора.

Для начала проведем конечно-разностную аппроксимацию уравнения (9)

$\rho(t+\Delta t)=U \rho(t) U^{\dagger}+\Delta t\left(\Gamma_{\varphi} \sigma_{z} \rho \sigma_{z}+\Gamma_{e} \sigma_{-} \rho \sigma_{+}+\gamma a \rho a^{\dagger}\right)$.

Можно видеть, что изменение матрицы плотности $\rho(t+\Delta t)=\sum_{i}\left|\phi_{i}(t+\Delta t)\right\rangle\left\langle\phi_{i}(t+\Delta t)\right|$ обусловлено дву- мя возможными вкладами, сопровождающимися изменением коэффициентов $C_{i, n, \sigma}(t)$ стохастической волновой функции $\left|\phi_{i}(t)\right\rangle$.

Первый вклад (первое выражение в формуле (10)) определяется оператором эволюции $U=e^{-i H_{\mathrm{dis}} \Delta t / \hbar}$ и отвечает за диссипативную динамику системы с гамильтонианом Вигнера-Вайскопфа

$$
H_{\mathrm{dis}}=H-i \hbar \frac{\Gamma_{\varphi}}{4} \sigma_{z}^{\dagger} \sigma_{z}-i \hbar \frac{\Gamma_{e}}{2} \sigma_{+} \sigma_{-}-i \hbar \frac{\gamma}{2} a^{\dagger} a
$$

При этом изменение волновой функции $\left|\phi_{i}(t)\right\rangle$ („траектории“ ) можно представить выражением

$$
\left|\phi_{i}(t+\Delta t)\right\rangle=U\left|\phi_{i}(t)\right\rangle / \sqrt{1-\Delta P},
$$

где введены следующие обозначения:

$$
\Delta P=\Delta t \bar{\gamma},
$$

$$
\bar{\gamma}=\Gamma_{\varphi}+\frac{\Gamma_{e}}{2}\left(1+\sum_{n, \sigma} \sigma\left|C_{i, n, \sigma}(t)\right|^{2}\right)+\gamma \sum_{n, \sigma} n\left|C_{i, n, \sigma}(t)\right|^{2} .
$$

Второе слагаемое в уравнении (10) можно связать со спонтанными переходами (скачками) в системе за счет взаимодействия с бозонным термостатом. Попытаемся придать данному слагаемому вероятностный смысл, имея в виду, что с уравнением (10) можно связать некоторый случайный процесс, который будет имитировать относительный вклад диссипативной динамики и спонтанных переходов, происходящих с вероятностью $\Delta P$, а изменение „траектории“ может сопровождаться в рассматриваемой системе одним из скачков: фазовым или энергетическим для кубита и спонтанным испусканием кванта для осциллятора. При этом данные процессы влияют на мгновенное (на интервале времени разрешения $\Delta t)$ изменение коэффициентов разложения $C_{i, n, \sigma}(t)$ для волновой функции $\left|\phi_{i}(t)\right\rangle$ :

в случае фазового скачка для кубита

$$
C_{i, n, \sigma}^{\varphi}(t+\Delta t)=\sigma C_{i, n, \sigma}(t),
$$

в случае энергетического скачка для кубита

$$
C_{i, n, \sigma}^{e}(t+\Delta t)=\frac{\sum_{\sigma^{\prime}}\left(\sigma_{-}\right)_{\sigma \sigma^{\prime}} C_{i, n, \sigma^{\prime}}(t)}{\sqrt{\frac{1}{2}\left(1+\sum_{n, \sigma} \sigma\left|C_{i, n, \sigma}(t)\right|^{2}\right)}},
$$

в случае энергетического скачка для осциллятора

$$
C_{i, n, \sigma}^{\gamma}(t+\Delta t)=\frac{\sqrt{n+1} C_{i, n+1, \sigma}(t)}{\sum_{n, \sigma} n\left|C_{i, n, \sigma}(t)\right|^{2}} .
$$

Коэффициенты разложения определены со следующими весами:

$$
\begin{gathered}
p_{\varphi}=\frac{\Gamma_{\varphi}}{\bar{\gamma}}, \quad p_{\varepsilon}=\frac{\Gamma_{e}}{2 \bar{\gamma}}\left(1+\sum_{n, \sigma} \sigma\left|C_{i, n, \sigma}(t)\right|^{2}\right), \\
p_{\gamma}=\frac{\gamma}{\bar{\gamma}} \sum_{n, \sigma} n\left|C_{i, n, \sigma}(t)\right|^{2} .
\end{gathered}
$$


Итак, решая уравнения для коэффициентов разложения волновой функции $C_{i, n, \sigma}(t)$, за время наблюдения эволюции системы мы получаем квантовую траекторию, представляющую одну реализацию мысленного эксперимента. Поскольку процесс релаксации является случайным, каждая траектория уникальна. Для нахождения усредненной динамики (аналог того, что получается в результате решения уравнения (9))следует получить набор реализаций $M \sim 1000-10000$ и усредненную матрицу плотности

$$
\rho=\frac{1}{M} \sum_{i=1}^{M}\left|\phi_{i}(t)\right\rangle\left\langle\phi_{i}(t)\right| .
$$

Можно вычислить ожидаемое значение любой наблюдаемой величины квантовой системы. Например, среднее число квантов осциллятора для двух состояний кубитов (основного , $\downarrow^{“}(\sigma=-1)$ и возбужденного , $\left.\uparrow “(\sigma=1)\right)$ составляет

$$
\begin{gathered}
\bar{n}_{\uparrow}=\frac{1}{M} \sum_{i=1}^{M} \sum_{n=0}^{N-1} n\left|C_{i, 2 n+1, \uparrow}(t)\right|^{2}, \\
\bar{n}_{\downarrow}=\frac{1}{M} \sum_{i=1}^{M} \sum_{n=0}^{N-1} n\left|C_{i, 2 n, \downarrow}(t)\right|^{2} .
\end{gathered}
$$

Отметим, что квантовый метод Монте-Карло по сравнению с прямым решением уравнения для матрицы плотности имеет ряд преимуществ. Во-первых, это возможность исследовать релаксационные процессы квантовых систем в единичных реализациях. Во-вторых, данный метод позволяет осуществить эффективное распараллеливание численного алгоритма. Поскольку реализации состояний статистически независимы, есть возможность генерировать каждую реализацию в отдельном потоке (на отдельном процессоре), собирая затем данные и производя усреднение.

\section{4. Временной мониторинг состояния кубита в единичных реализациях и в среднем при подаче Раби-импульса}

При изучении процесса детектирования состояния кубита измерительным осциллятором важную роль играет соотношение между собственной частотой кубита $\omega_{q}$ и плазменной частотой джозефсоновского измерительного осциллятора $\omega_{J}$. Предварительные численные эксперименты по моделированию систем с различными энергетическими параметрами показали, что если $\omega_{q} \approx \omega_{J}$, то при записи информации на кубит Раби-импульсом с частотой $\omega$ происходит процесс сильной перекачки энергии между подсистемами, что усложняет процесс записи, а также усиливает эффект обратного действия (влияние измерительного прибора на состояние кубита). Когда частоты кубита и осциллятора сильно различаются, процесс измерения состояний кубита будет

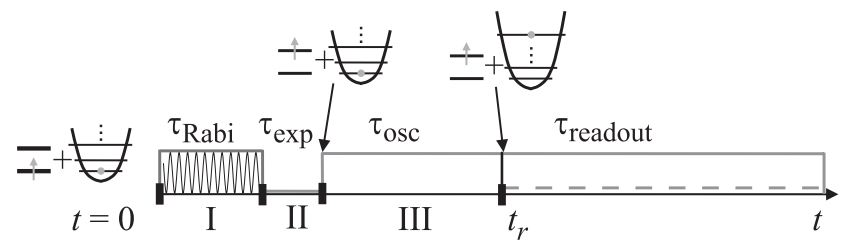

Рис. 2. Временна́я развертка последовательности подачи импульсов на систему кубит-осциллятор. Область I - управляющий импульс записи $\tau_{\text {Rabi, }}$ область II - время ожидания $\tau_{\text {exp }}$, область III соответствует импульсу „запитки“ измерительного сигнала $\tau_{\text {osc }}$, а штриховая прямая (начиная с $t_{r}$ ) - считыванию информации $\tau_{\text {readout. }}$.

затруднен ввиду слабой связи между подсистемами. Таким образом, исследования показали, что наилучшим для проведения измерений является диапазон частот, определяемый соотношением $3<\omega_{q} / \omega_{J}<12$, что соответствует экспериментальным данным [1].

На основе экспериментов по неразрушающему измерению сверхпроводниковых кубитов [1-3], была предложена схема считывания состояний кубита на базе импульсного воздействия, представленная на рис. 2. Предварительно проводится инициализация системы, когда кубит и осциллятор приготовлены в основных состояниях. При этом в начальный момент времени волновая функция системы для $i$-й реализации выбирается в виде

$$
\left|\phi_{i}(0)\right\rangle=\sum_{n=0}^{N} \sum_{\sigma=1}^{2} C_{i, n, \sigma}(0)|n \sigma\rangle,
$$

где $C_{i, n, \sigma}(0)=0$, кроме $C_{i, 0,1}(0)=1$. Далее на кубит подается Раби-импульс записи заданной длительности $\tau_{\mathrm{Rabi}}=2 \pi / \Omega_{\mathrm{Rabi}} \quad\left(\Omega_{\mathrm{Rabi}}=\sqrt{\left(\omega_{q}-\omega\right)^{2}+A^{2}}-\right.$ частота Раби). Естественно, за счет связи между подсистемами (кубит и измерительный осциллятор) после окончания действия импульса записи существует ненулевая вероятность возбуждения осциллятора. Хотя можно подобрать такие параметры системы, чтобы в результате осциллятор возбуждался лишь вблизи основного состояния. В силу этого для установления переходных процессов между подсистемами до момента подачи управляющего поля $\tau_{\text {osc }}$ введено время ожидания $\tau_{\exp }$ (область II на рис. 2). Как показали численные эксперименты, длительность времени ожидания, достаточная для того, чтобы осциллятор находился вблизи основного состояния с вероятностью $99 \%$, составляет $\tau_{\text {exp }} \sim 0.5 \tau_{\text {Rabi. }}$. После этого на измерительный прибор подается импульс „запитки“ длительностью $\tau_{\text {osc }}$ (область III на рис. 2), который возбуждает осциллятор. К моменту времени $t_{r}$ он захватывается в квантовый нелинейный резонанс и начинается действие считывающего импульса $\tau_{\text {readout }}$ (на рис. 2 соответствующая область показана штриховой линией). При этом снимаются показания с измерительного осциллятора, находящегося вблизи точки бифуркации при мезоскопическом режиме работы (число квантов составляет несколько десятков). 

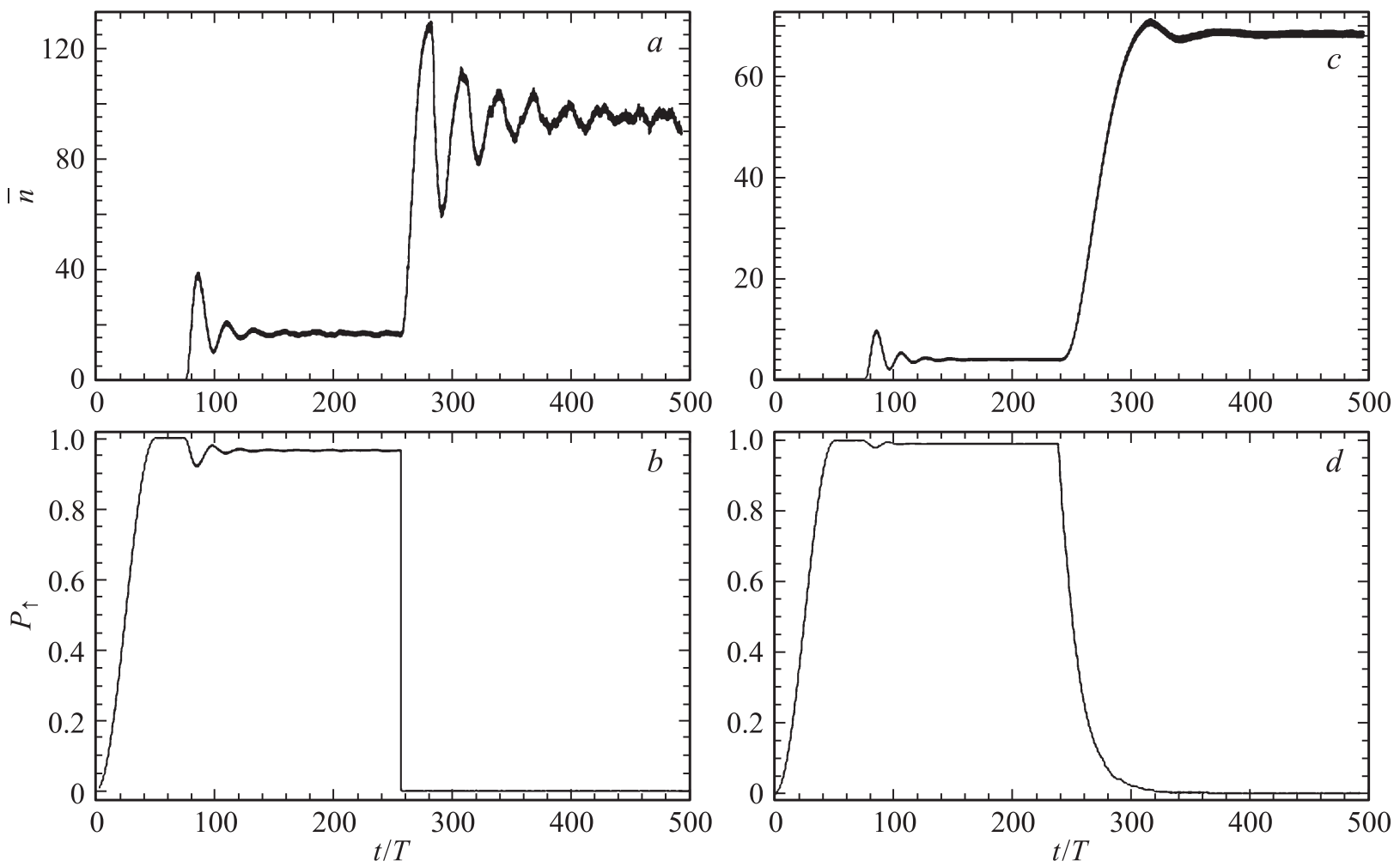

Рис. 3. Одна реализация временно́го мониторинга населенностей нелинейного осциллятора $\bar{n}(a)$ и вероятности нахождения кубита на верхнем уровне $P_{\uparrow}(b)$; усредненная по $M=10000$ реализациям временна́я динамика населенностей нелинейного осциллятора $\bar{n}(c)$ и вероятности нахождения кубита на верхнем уровне $P_{\uparrow}(d)$. Параметры системы и шума: $\omega_{q}=6 \omega_{J}, \omega=6.01 \omega_{J}$, $A=0.02 \hbar \omega_{J}, f_{0}=0.2 \hbar \omega_{J}, \Omega=1.02 \omega_{J}, \mu=0.0001 \hbar \omega_{J}, \lambda=0.02, \Gamma_{e}=0.00065 \hbar \omega_{J}, \Gamma_{\varphi}=0.001 \hbar \omega_{J}$ и $\gamma=0.01 \hbar \omega_{J}$.

В случае $\pi$-импульса записи осциллятор позволяет произвести неразрушающие измерения состояний кубита, так как после записи информации гамильтониан кубита коммутирует с гамильтонианом измерительного прибора. Благодаря данному факту возможно извлечение информации о состоянии кубита в каждой уникальной реализации - аналог „однократных“ измерений [3]. Квантовым методом Монте-Карло в соответствии со схемой подачи импульсов, изображенной на рис. 2, промоделировано поведение населенности возбужденного уровня кубита и изменение числа фотонов для единичной реализации $(M=1)$, (рис. $3, a, b)$.

Из анализа единичных траекторий, аналогичных тем, что изображены на рис. $3, a, b$, следует, что после начала воздействия внешнего поля на осциллятор вероятность возбужденного уровня кубита испытывает небольшие колебания, что соответствует эффекту обратного действия. Численные эксперименты показали, что перекачка энергии между подсистемами составила примерно $1 \%$ для параметров системы, соответствующих рис. $3, a, b$. Выяснено, что чем больше амплитуда внешней силы (тока) для осциллятора, тем сильней эффект обратного действия. Так, например, для $f_{0}=0.4 \hbar \omega_{J}$ перекачка энергии между подсистемами составляет уже $5 \%$. Спустя некоторое время установления $\tau_{\text {osc }} \sim 100 T_{0}\left(T_{0}=2 \pi / \Omega\right)$ осциллятор захватывается в нелинейный резонанс вблизи некоторой группы уровней, что соответствует услов- но среднему значению населенностей $\overline{n_{\uparrow}}$ для возбужденного состояния кубита.

Как известно, основным параметром, влияющим на спонтанное изменение состояния кубита, является нарушение когерентности в системе, связанное с энергетическим шумом. В случайный момент времени, определяемый характерным временем энергетической релаксации $\tau_{e}=1 / \Gamma_{e}$, кубит мгновенно (за время, меньше предела временно́го разрешения экспериментальной установки) переходит из возбужденного состояния в основное (например, в случайной реализации, изображенной на рис. $3, a, b$, скачок произошел при $t=260 T$ ). При этом кубит передает квант энергии осциллятору, работающему в мезоскопическом режиме вблизи точки бифуркации, что неминуемо влияет на изменение его числа фотонов. На бифуркационной кривой для нелинейного осциллятора это соответствует „перебросу“ с одной резонансной кривой на другую $\left(\bar{n}_{\uparrow} \rightarrow \bar{n}_{\downarrow}\right)$ при фиксированной частоте и амплитуде внешнего поля. Таким образом, в эксперименте, следя только за показаниями измерительного прибора (во время действия импульса $\tau_{\text {readout }}$ ), можно восстановить информацию о состоянии кубита в реальном времени. Следовательно, бифуркационный осциллятор может выступать в роли детектора квантовых скачков для мониторинга состояний системы в реальном времени и реализации „однократных“ измерений. 
Квантовый метод Монте-Карло может быть использован при моделировании результатов, наблюдаемых в экспериментах по проективным измерениям $[1,2]$, т. е. динамики системы, усредненной по реализациям. В этом случае проводится $M \sim 10000$ повторений опытов согласно схеме подачи управляющих импульсов, изображенной на рис. 2. При этом погрешность за счет использования стохастического метода зависит от числа повторений и составляет $\sim 1 / \sqrt{M}=1 \%$. Момент квантового скачка населенности кубита (сброса в основное состояние) случайный процесс на временах, близких к $\tau_{e}=1 / \Gamma_{e}$, что на усредненной зависимости населенности верхнего уровня кубита от времени (рис. 3,d) выглядит пологим спуском, характеризуемым временем энергетической релаксации, в отличие от резкого вертикального срыва в индивидуальном скачке (рис. $3, b$ ). Плавное изменение состояния кубита (эволюция в основное состояние) отражается и в „затянутости“ выхода осциллятора на установившееся усредненное значение $\bar{n}_{\downarrow}$ (рис. $3, c$ ). По сравнению с одной реализацией усредненные характеристики являются более гладкими за счет усреднения по случайным реализациям. Скорость энергетической релаксации определяет быстроту перехода кубита из одного базисного состояния в другое. С увеличением скорости энергетических потерь в кубите переход в основное состояние становится более резким. Фазовый шум в данных экспериментах не оказывает сильного влияния на кубит, так как происходит лишь сбой фазы волновой функции, а энергия системы не изменяется.

При анализе информации, считываемой с измерительного осциллятора, можно изучить разброс населенностей по уровням, чтобы понять, в каком состоянии $(\downarrow-$ основное состояние, $\uparrow-$ возбужденное) и с какой вероятностью находится кубит. Для этого с момента начала действия считывающего импульса $t_{r}$ (рис. 2) необходимо хранить инофрмацию о состоянии осциллятора для каждой квантовой траектории. После сбора необходимых статистических данных можно построить гистограммы распределения населенностей и проследить, как кубит переходит из возбужденного состояния в основное. Прямоугольные столбики на гистограмме (рис. 4) отражают, какое количество отсчетов для всех „квантовых траекторий“ попало в выделенный интервал населеенностей. На рис. 4 приведены четыре гистограммы распределения вероятностей для различных моментов времени. Для случая, когда кубит находится в возбужденном состоянии (рис. 4, $a$ ), видно, что гистограмма распределения населенностей сосредоточена около значения $\bar{n}_{\uparrow} \sim 3.93$ (центр распределения), а сплошная кривая - аппроксимирующая функция распределения - построена по статистическим данным для $M=10000$ реализаций. Ширина распределения $\delta \bar{n}^{\uparrow}$ (основание гистограммы) лежит в интервале $3.85 \leq \delta \bar{n}_{\uparrow} \leq 4.03$. Все квантовые траектории попали именно в этот энергетический интервал, что свитедельствует о нахождении кубита на верхнем уровне с вероятностью 100\%. Естественно, что ширины функций распределений для основного и
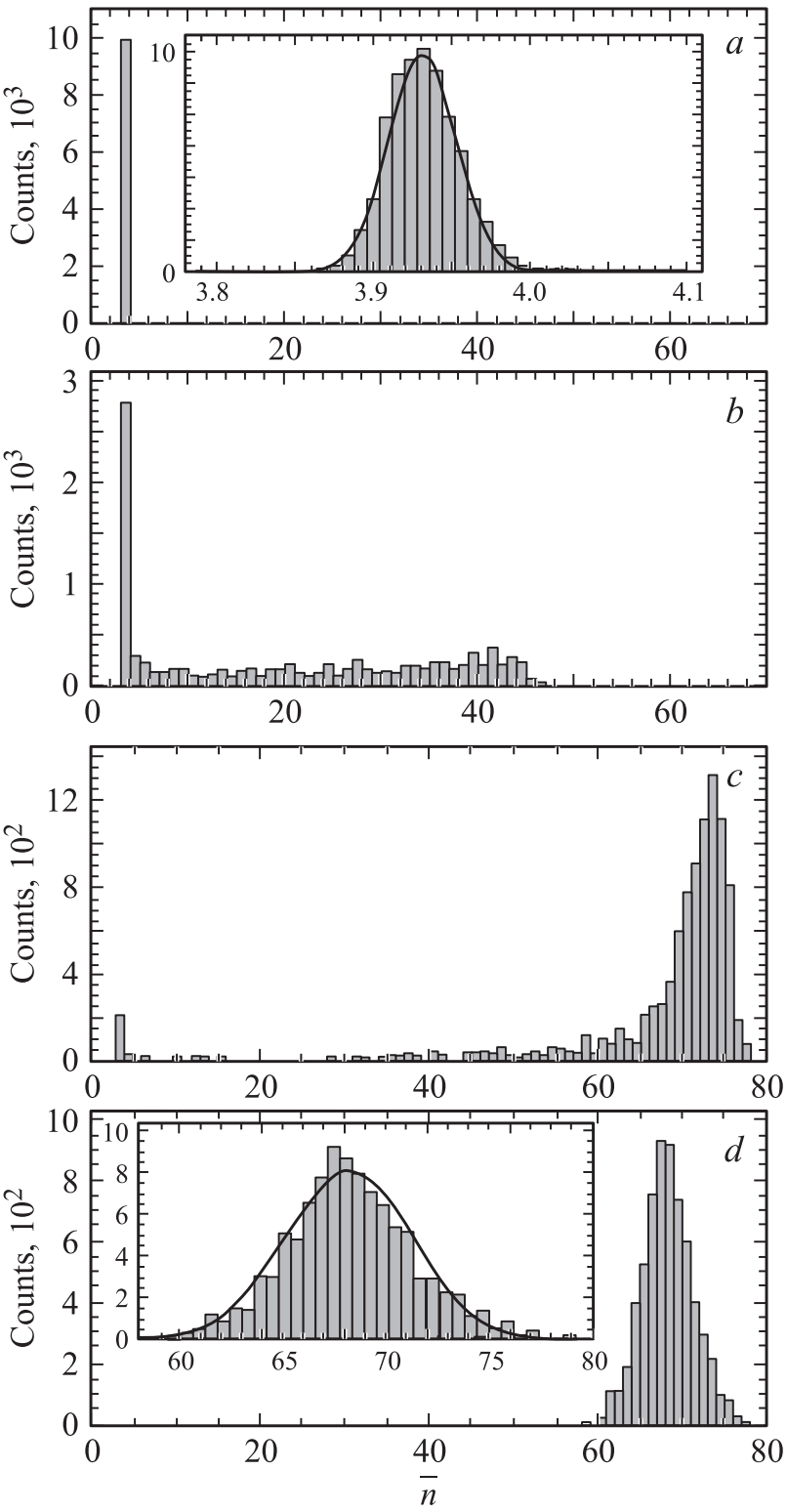

Рис. 4. Гистограммы распределения населенностей осциллятора в системе, представленной на рис. 3, $c$, для моментов времени $t=200 T(a), 260 T(b), 280 T(c)$ и $460 T(d)$. Число реализаций $M=10000$.

возбужденного состояний кубита различны, так как чем выше по энергии возбуждается осциллятор, тем бо́льшая группа уровней входит в нелинейный резонанс с частотой осциллятора. Ширина распределения для основного состояния кубита $55 \leq \delta \bar{n}_{\downarrow} \leq 80$ (рис. $4, d$ ).

Для переходного временно́го интервала, когда имеет место пологий спад и происходит перераспределение вероятностей населенностей уровней кубита, приведены две гистограммы (рис. 4, $b, c)$. Видно, что до момента времени $t=260 T$ (рис. 4, $a, b$ ) более вероятно возбужденное состояние кубита, а начиная с $t=280 T$ (рис. $4, c, d$ ) кубит находится с большей вероятностью в основном состоянии. Подобное разделение состояний 


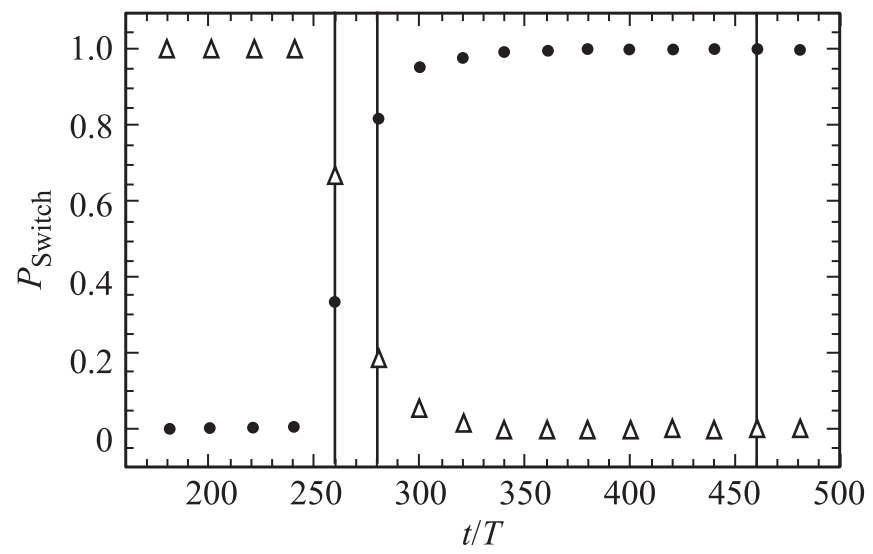

Рис. 5. Вероятность переключения $P_{\text {Switch для возбужденного }}$ (треугольники) и основного (точки) состояний кубита. Параметры системы те же, что для рис. $3, c, d$.

кубита возможно, так как населенности уровней осциллятора, соответствующих базисным уровням кубита, хорошо разнесены по числу квантов (функции распределения не перекрываются) и в момент энергетического скачка кубита населенности уровней осциллятора изменятся на значительную величину: $\Delta \bar{n}=\bar{n}_{\downarrow}-\bar{n}_{\uparrow} \sim 50$ (интервал между центрами распределений базисных состояний), что и регистрируется в эксперименте.

Кроме того, можно ввести вероятность переключения $P_{\text {Switch, }}$ характеризующую состояние кубита по числу квантов осциллятора, которая показывает долю квантовых траекторий, попавших либо в интервал энергий $\delta \bar{n}_{\uparrow}$ (треугольники на рис. 5), либо в интервал $\delta \bar{n}_{\downarrow}$ (точки на рис. 5). Вертикальные прямые на рис. 5 показывают моменты времени, отраженные на гистограммах распределения населенностей осциллятора (рис. 4). Ана- логичная величина измеряется в экспериментах $[1,3]$, но не для временно́го мониторинга состояний кубита, а для сканирования по внешнему току.

\section{5. Временной мониторинг суперпозиционного состояния кубита}

В этом разделе описывается применение изложенной ранее методики „дискриминирования“ состояния кубита по измерению вероятностей переключения $P_{\text {Switch }}$ населенностей нелинейного осциллятора, когда кубит находится в суперпозиционном состоянии. Для получения такого состояния можно использовать Раби-импульсы различной длительности $\tau_{\mathrm{Rabi}}$, в момент прекращения действия которых кубит находится с вероятностью $P_{\downarrow}$ на нижнем уровне и с вероятностью $P_{\uparrow}$ на верхнем. Для демонстрации данной техники измерения было промоделировано действие Раби-импульсов длительностью $\tau_{\text {Rabi }}=0.3 \frac{2 \pi}{\Omega_{\text {Rabi }}}($ рис. 6, $a, b), \tau_{\text {Rabi }}=0.5 \frac{2 \pi}{\Omega_{\text {Rabi }}}($ рис. 6, $c, d)$ и $\tau_{\text {Rabi }}=0.8 \frac{2 \pi}{\Omega_{\text {Rabi }}}$ (рис. 6,e,f). При этом импульсное воздействие полностью аналогично представленному на рис. 2, а измеряемые величины (населенность осциллятора и вероятность нахождения кубита на верхнем уровне) усреднены по $M=10000$ квантовым реализациям. В начальный момент действия импульса возбуждения измерительного прибора $\tau_{\text {osc }}$, как и для случая $\pi$-импульса, происходит процесс обратного действия. Далее осциллятор выходит на некоторое установившееся значение $\bar{n}$, которое соответствует суперпозиции состояний $\bar{n}_{\uparrow}$ и $\bar{n}_{\downarrow}$, а доля содержания каждого из них характеризуется величиной $P_{\text {Switch }}$, представленной на рис. 7.
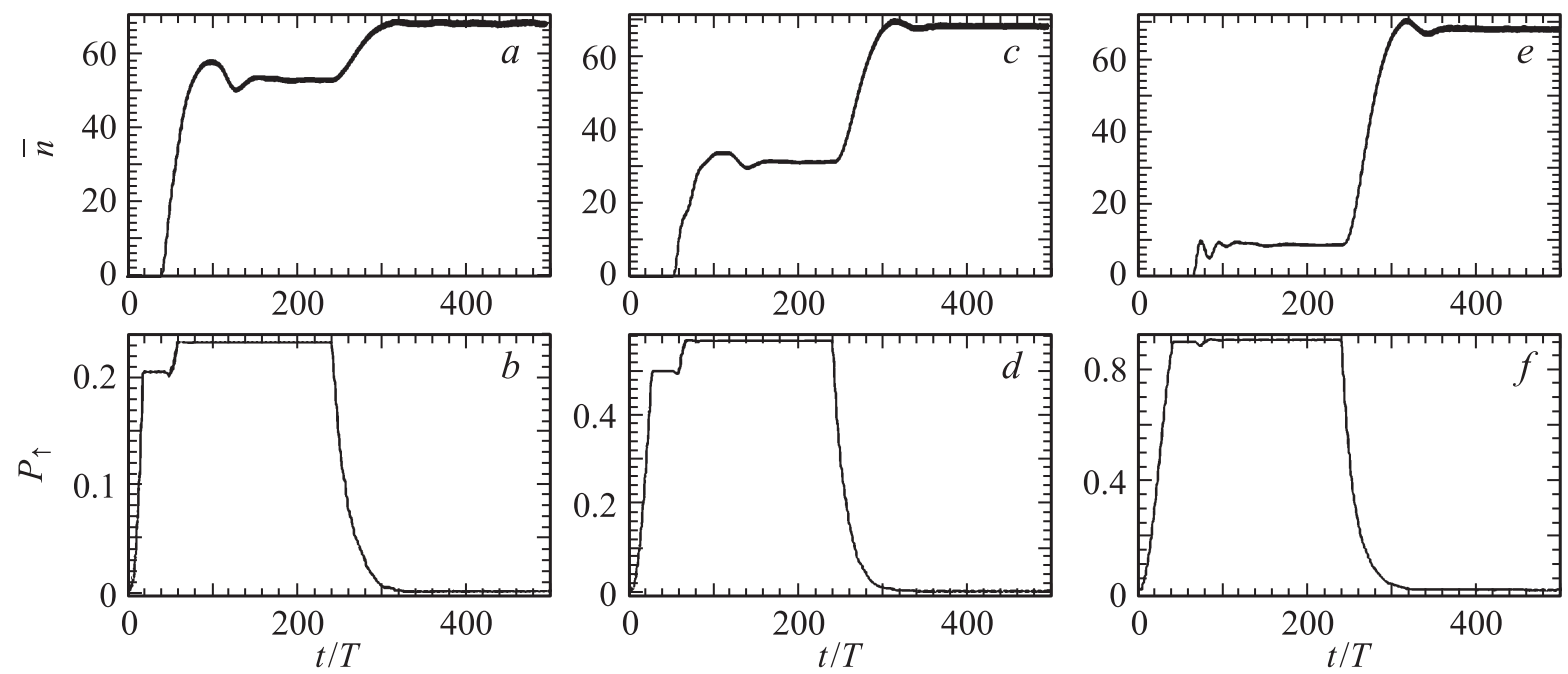

Рис. 6. Усредненная по $M=10000$ реализациям динамика населенностей нелинейного осциллятора $\bar{n}(a, c, e)$ и вероятности нахождения кубита на верхнем уровне $P_{\uparrow}(b, d, f)$ для различных значений длительности Раби-импульса: $\tau_{\text {Rabi }}=0.3 \frac{2 \pi}{\Omega_{\mathrm{Rabi}}}(a, b)$, $0.5 \frac{2 \pi}{\Omega_{\text {Rabi }}}(c, d)$ и $0.8 \frac{2 \pi}{\Omega_{\text {Rabi }}}(e, f)$. Параметры системы те же, что для на рис. $3, a$. 

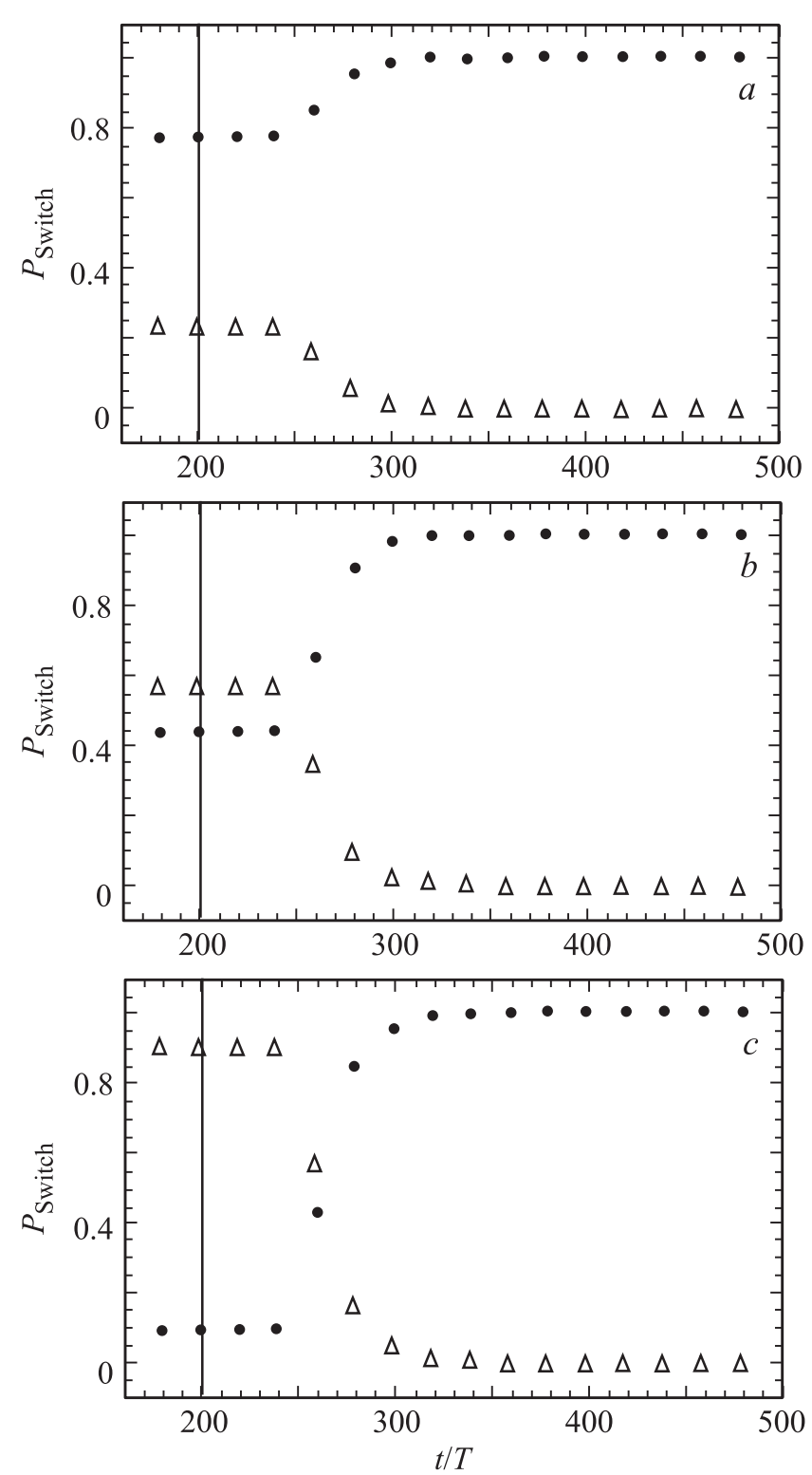

Рис. 7. Вероятность переключения $P_{\text {Switch для возбужденного }}$ (треугольники) и основного (точки) состояний кубита при разной длительности импульсов записи: $\tau_{\text {Rabi }}=0.3 \frac{2 \pi}{\Omega_{\text {Rabi }}}(a)$, $0.5 \frac{2 \pi}{\Omega_{\text {Rabi }}}(b)$ и $0.8 \frac{2 \pi}{\Omega_{\text {Rabi }}}(c)$. Параметры системы те же, что для рис. $3, a$.

Отметим, что благодаря такому способу считывания состояний кубита можно из экспериментальных численных данных найти долю обратного действия измерительного прибора на кубит, подавая импульс заданной длительности и рассчитав вероятность нахождения кубита в возбужденном состоянии. Например, для Рабиимпульса $\tau_{\text {Rabi }}=0.5 \frac{2 \pi}{\Omega_{\text {Rabi }}}$ (импульс $\left.\pi / 2\right)$ населенности верхнего и нижнего уровней кубита будут равны. Однако в результате измерений видно (рис. 7), что населенность верхнего уровня до момента энергетической релаксации $(180 T \leq t \leq 240 T)$ равна $P_{\uparrow}=0.57$; следовательно, процесс передачи энергии от осциллятора к кубиту составил 7\%. Аналогично можно провести расчеты и для других длительностей импульса.

Отметим, что временно́й мониторинг суперпозиционного состояния кубита в одной реализации указанным способом подачи сигналов не является информативным. Это связано с тем, что подсистемы являются сильно скоррелированными, и измерительный прибор в момент возбуждения будет передавать энергию кубиту, что может привести к полному возбуждению кубита.

\section{6. Заключение}

В работе на основе моделирования динамики системы кубит-измерительный осциллятор в бозонном термостате предложен способ временно́го мониторинга состояний кубита нелинейным осциллятором, работающим в мезоскопическом режиме, когда среднее число квантов хотя и велико, но конечно, а среднеквадратичное отклонение мало по сравнению с числом заполнения. Показано, что для заданной длительности импульса записи можно определить относительную величину влияния измерительного прибора на состояния квантовой системы (эффект обратного действия). Результаты численного моделирования позволили выявить, что с помощью нелинейного осциллятора можно проводить измерения, подобные „однократным“ в реальном времени для $\pi$-импульса записи, и наблюдать отдельные квантовые скачки. Для усредненной динамики системы на основе накопления статистических данных изучена эволюция плотности распределения средней энергии осциллятора, которая показывает вероятность нахождения кубита в том или ином базисном состоянии. Данный численный подход позволяет моделировать процесс „распутывания“ суперпозиционного состояния кубита по принципу неразрушающих проекционных измерений.

\section{Список литературы}

[1] I. Siddiqi, R. Vijay, M. Metcalfe, E. Boaknin, L. Frunzio, R.J. Schoelkopf, M.H. Devoret. Phys. Rev. B 73, 054510 (2006).

[2] T. Picot, A. Lupascu, S. Saito, C.J.P.M. Harmans, J.E. Mooij. Phys. Rev. B 78, 132508 (2008).

[3] R. Vijay, D.H. Slichter, I. Siddiqi. Phys. Rev. Lett. 106, 110502 (2011).

[4] М.О. Скалли, М.С. Зубайри. Квантовая оптика. Физматлит, M. (2003). $510 \mathrm{c}$.

[5] M.B. Plenio, P.L. Knight. Rev. Mod. Phys. 70, 101 (1998).

[6] D. Vion, A. Aassime, A. Cottet, P. Joyez, H. Pothier, C. Urbina, D. Esteve, M.H. Devoret. Science 296, 886 (2002)

[7] V.B. Braginsky, F.Ya. Khalil. Quantum measurement. Cambridge University Press, Cambridge (1995). 191 p. 